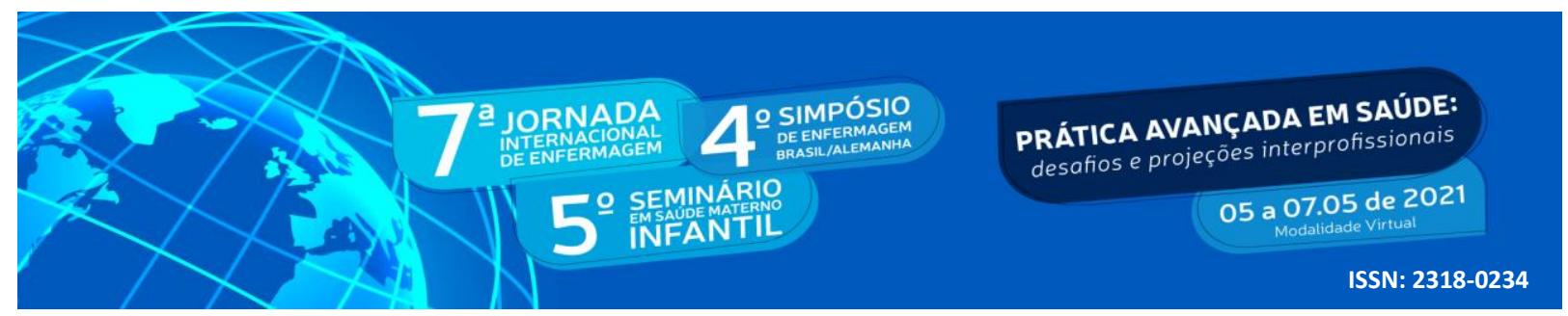

DOI: http://doi.org/10.48195/jie2021-080

\title{
O TRABALHO EM EQUIPE MULTIPROFISSIONAL SOB A ÓTICA DA ENFERMAGEM: UM RELATO DE EXPERIÊNCIA ${ }^{1}$
}

\author{
Victória Friedrich da Costa²; Leandro da Silva de Medeiros ${ }^{3}$; Giovana Luiza Rossato ${ }^{4}$; \\ Graziele Alves Flores ${ }^{5}$; Aline Dalcin Segabinazi ${ }^{6}$; Camila dos Santos Gonçalves ${ }^{7}$
}

\begin{abstract}
RESUMO
Objetivo: Refletir acerca dos desafios do trabalho em equipe multiprofissional sob a ótica da enfermagem. Método: Trata-se de um estudo descritivo, do tipo relato de experiência, desenvolvido por discentes do curso de Enfermagem no $2^{\circ}$ semestre de 2020 provocado por um trabalho na disciplina Psicologia e Saúde. Realizou-se um levantamento bibliográfico e um formulário com profissional da área, a partir do preenchimento de um formulário on-line no período de outubro à dezembro de 2020, no decorrer da disciplina de Psicologia e Saúde. Resultados: Foi delimitado dois pontos chaves de análise: espaço para planejamento e ações conjuntas e comunicação entre profissionais. Conclusão: Considera-se que para a equipe multiprofissional poder atuar de forma coesa e sistematizada é preciso manter espaços de trocas dialógicas sistemáticas, pois os desafios e dificuldades emergem no cotidiano de trabalho e podem permear as relações de trabalho se não houver uma boa comunicação interna entre a equipe.
\end{abstract}

Palavras-chave: Equipe de Assistência ao Paciente; Enfermagem; Integralidade em Saúde; Serviços de Saúde.

\section{ABSTRACT}

Objective: To reflect on the challenges of multiprofessional teamwork from the perspective of nursing. Method: This is a descriptive study, of the type of experience report, developed by students of the nurs ing course in the 2nd semester of 2020 provoked by a work in the discipline Psychology and Health. A bibliographic survey and an interview were conducted with a professional in the area, from the com pletion of an online form from October to December 2020, during the psychology and health course. Results: Two key points of analysis were delimited: space for planning and joint actions and communi cation between professionals. Conclusion: It is considered that for the multidisciplinary team to be able to act in a cohesive and systematized is necessary to maintain spaces of systematic dialogical,because challenges and difficulties emerge in daily work and can permeate work relationships if there is no go od internal communication between the team.

\footnotetext{
1 Trabalho avaliativo apresentado no $2^{\circ}$ semestre de 2020 na disciplina Psicologia e Saúde do Curso de Enfermagem, Universidade Franciscana

2 Estudante do Curso de Enfermagem, Universidade Franciscana. E-mail: victoriafriedrich27@gmail.com

3 Estudante do Curso de Enfermagem, Universidade Franciscana. E-mail: leandro.medeiros@ufn.edu.br

4 Estudante do Curso de Enfermagem, Universidade Franciscana. E-mail: rossatogiovana@gmail.com

5 Estudante do Curso de Enfermagem, Universidade Franciscana. E-mail: flores.graziele8@gmail.com

6 Enfermeira pela Universidade Franciscana. Mestre em Saúde Materno Infantil. E-mail: alinesegabinazi@gmail.com

7 Psicóloga. Professora responsável pela disciplina em 2/2020. E-mail: camila.gon.79@gmail.com
} 


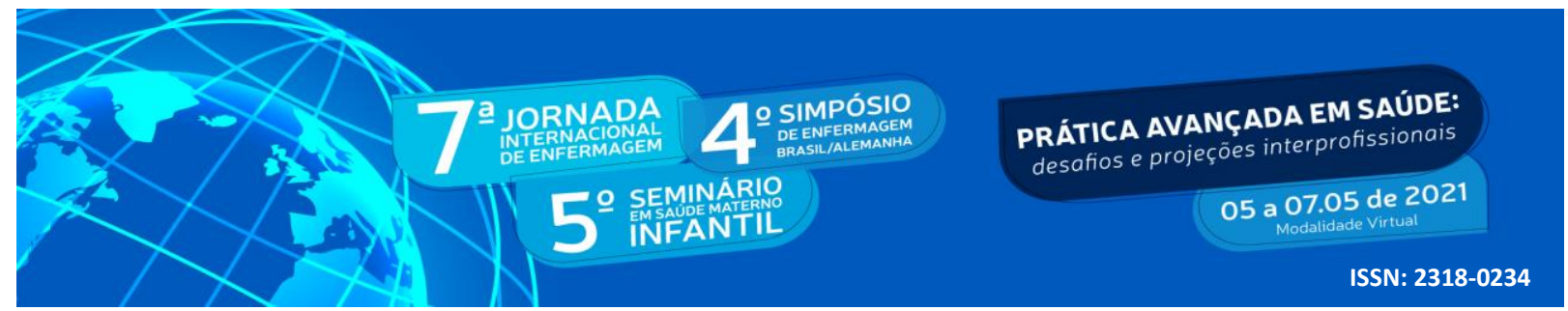

Key Words: Patient Care Team; Nursing; Integrality in Health; Health Services.

\section{INTRODUÇÃO}

Na equipe multiprofissional, a articulação refere-se à recomposição de processos de trabalhos distintos e a consideração de conexões e interfaces, entre as intervenções técnicas peculiares de cada área profissional. Trabalhar em equipe de modo integrado significa conectar diferentes processos de trabalho, com base no conhecimento do trabalho do outro e valorização da participação deste na produção de cuidados (PEDUZZI; AGRELI, 2018).

A importância desta equipe no serviço de saúde dá-se por meio da agregação de conhecimentos em prol de um cuidado integral ao paciente, seja ele em atendimento primário ou nível complexo. O trabalho em equipe amplia a visão do processo de saúde, envolvendo diferente saberes e intervindo, para além do âmbito individual e clínico, na família e nas condições socioambientais da comunidade atendida (BACKES et al., 2014).

A ausência ou dificuldade de desenvolver a reflexão e diálogos entre os membros da equipe multiprofissional aproxima o trabalho da lógica fundamentada tanto em uma separação das disciplinas quanto dos seus objetos de estudo e intervenção. Consequentemente, uma sensação de dissociação e desagregação pode acometer o trabalhador, chegando ao usuário, pois, apesar de um discurso ser pautado pelo conceito de integralidade, a prática na assistência é fracionada e, muitas vezes, fragmentadora de processos e até de sujeitos (ANJOS FILHO; SOUZA, 2017, p. 72)

O trabalho multiprofissional está relacionado a uma união de diferentes áreas disciplinares que constituem as equipes, sendo, assim, referência de multidisciplinaridade, porém, orienta a uma pluridisciplinaridade pela pontuação de uma relação entre integração de diferentes conhecimentos. Contudo, um trabalho multiprofissional abrange toda a interação entre os participantes da equipe, assim, relacionando tanto à pluridisciplinaridade quanto à interdisciplinaridade (LANÇA; AMARAL; GRACIOSO, 2018).

As contribuições da enfermagem na equipe multiprofissional são refletidas em ações no qual favorecem o ingresso das pessoas dentro dos campos de saúde. Entre outras atuações do enfermeiro, também são consideradas no que diz respeito aos cuidados administrativos de cada lugar. Desse modo, a manutenção de equipamentos, reparos de materiais, organização e a limpeza das unidades assistenciais podem ser ponderadas pelos profissionais da enfermagem. Além disso, a relação ativa com todos os integrantes da equipe de saúde possibilita que o 


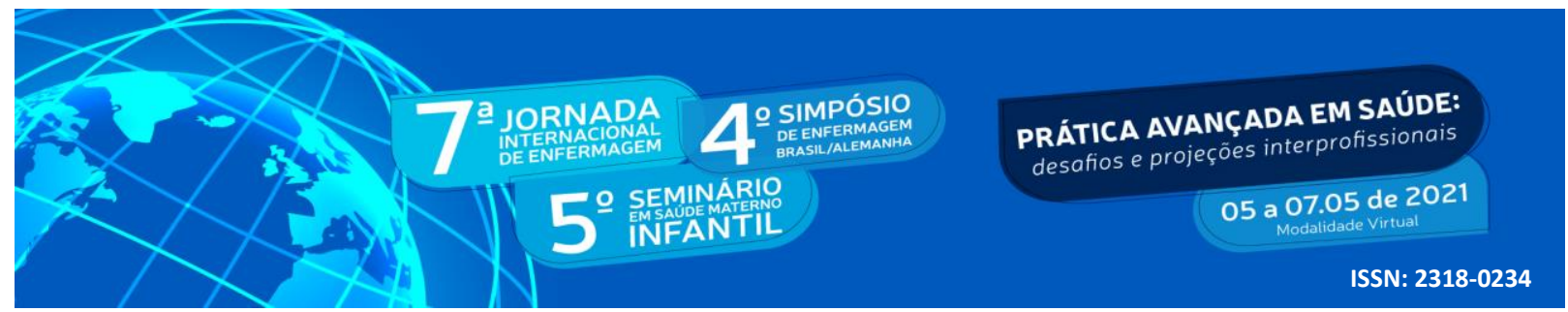

enfermeiro seja um fornecedor ativo de informações relacionadas aos pacientes (ALVES; SANTOS; LOPES, 2019).

No que diz respeito a liderança da enfermagem dentro das equipes é notória como são os dirigentes de orientações técnicas, transmissores de conhecimentos essenciais para as condutas e treinador das atividades realizadas dentro dos serviços. Nesse sentido, o papel da enfermagem dentro das equipes pode possibilitar uma melhora em todos os níveis de atenção, satisfazendo maior organização e segurança ao local de trabalho (CRUZ; ARAÚJO; BEZERRA, 2020).

Diante do exposto, considerando a importância do trabalho multiprofissional e a atuação do enfermeiro no trabalho em equipe, surge o seguinte questionamento: Quais são os desafios do trabalho em equipe multiprofissional sob a ótica da enfermagem?

\section{OBJETIVO}

Este estudo tem por objetivo refletir acerca dos desafios do trabalho em equipe multiprofissional sob a ótica da enfermagem.

\section{METODOLOGIA}

Trata-se de um estudo descritivo, do tipo relato de experiência, desenvolvido por quatro discentes do quinto semestre do curso de Enfermagem de uma instituição de ensino superior da região central do Rio Grande do Sul. O estudo ocorreu no período de outubro a dezembro de 2020, para a disciplina de Psicologia e Saúde do curso de graduação em Enfermagem, sob orientação da professora responsável.

O relato de experiência é uma modalidade de cultivo de conhecimento no território da pesquisa qualitativa, concebida na reinscrição e na elaboração ativada através de trabalhos da memória, em que o sujeito cognoscente implicado foi afetado e construiu seus direcionamentos de pesquisa ao longo de diferentes tempos. Isso posto, conjugará seu acervo associativo agindo processualmente, tanto em concomitância com o evento, como trazendo o produto processado pelas elaborações e em suas concatenações, e, finalmente, apresentará algumas das suas compreensões a respeito do vivido (DALTRO; FARIA, 2019). 


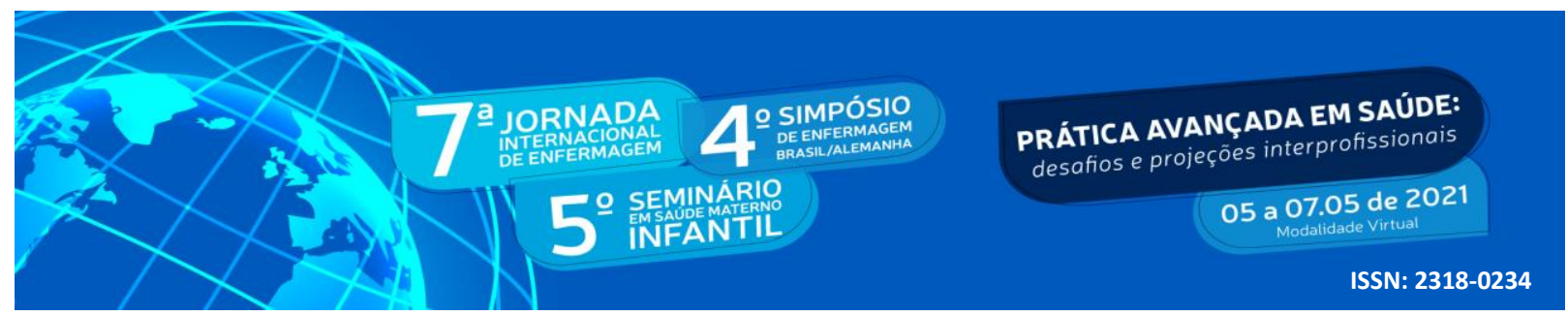

Nesse sentido, o trabalho foi realizado em grupo que buscou uma profissional atuante em uma Estratégia de Saúde da Família (ESF), situada em uma cidade do interior na região central do Rio Grande do Sul. Por conta da pandemia da Covid-19, foi disponibilizado um

formulário elaborado no Google Forms, contendo questões que exploravam suas percepções e experiências do trabalho em equipe no serviço de saúde. Tais estas: "quais são suas percepções no trabalho em equipe?”; "quais potencialidades e desafios que você percebe no trabalho em equipe?”; “ como você realiza o trabalho em equipe", e entre outras.

Ressalta-se, que o formulário para o trabalho atende ao $8^{\circ}$ ítem do artigo $1^{\circ}$ da Resolução 510/16 (BRASIL, 2016), em que diz que "atividade realizada com o intuito exclusivamente de educação, ensino ou treinamento sem finalidade de pesquisa científica" está dispensada de passar pela avaliação do Comitê de Ética em Pesquisa. Nesse sentido, os resultados desse estudo buscam relacionar experiências teórico-práticas para embasar a formação na área da saúde.

\section{RESULTADOS E DISCUSSÃO}

Após o grupo realizar a leitura do material bibliográfico foi possível conhecer um pouco da realidade e desafios do trabalho em equipe. Ao entrar em contato com a profissional convidada e conhecer um pouco da sua narrativa sobre o trabalho em equipe foi possível delimitar alguns pontos chaves de análise, são eles: Espaço para planejamento e ações conjuntas e Comunicação entre profissionais.

O campo de trabalho analisado para a reflexão foi uma Estratégia de Saúde da Família (ESF), identificou-se que as demandas do serviço surgem de modo espontâneo por queixas agudas, e logo encaminhadas para o acolhimento de enfermagem. Como foco principal a Unidade atende os/as usuários/as com agendamento prévio que tenham diagnóstico de doenças crônicas não transmissíveis, como diabetes e hipertensão, consulta de pré-natal de risco habitual e outras. Além disso, realiza atividades como vacinação, realização de curativos, administração de medicação, visitas domiciliares, e realização de atividades do programa saúde nas escolas.

Com a criação do Programa de Saúde da Família, mais tarde denominado de Estratégia Saúde da Família (ESF), no ano de 1994, este caráter circular e sistêmico do SUS ficou ainda mais evidente e necessário. Ao ser implementada como estratégia para a reorientação das práticas de saúde e, sobretudo, para a consolidação da atenção primária, no Brasil, a ESF requer, por excelência, processos altamente interativos e associativos com o propósito de apreender às necessidades de saúde do ponto de vista singular e multidimensional, ou seja, a partir dos múltiplos determinantes sociais, econômicos, políticos e outros (BACKES et al, 2014, p. 278). 


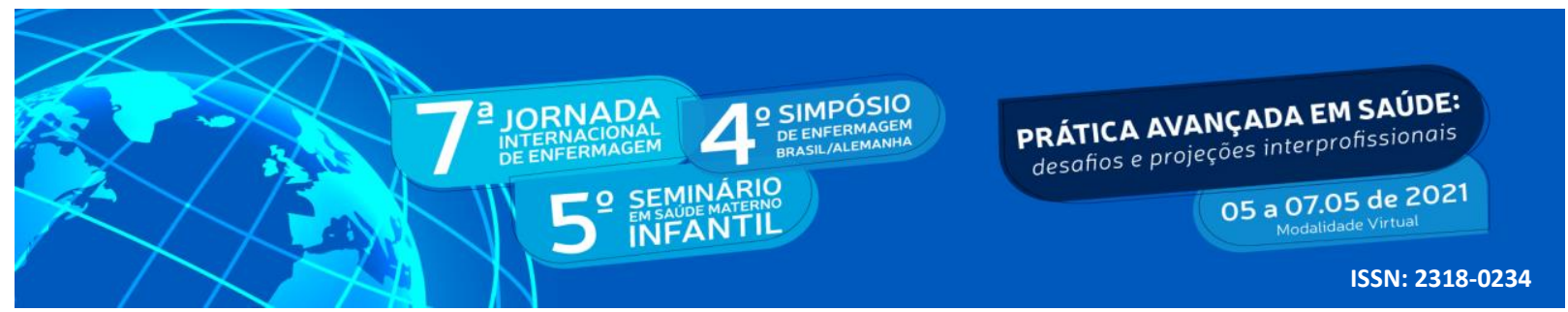

A Unidade tem duas equipes mínimas, compostas por profissionais das áreas da enfermagem (enfermeiro/a e técnico/a de enfermagem), medicina, e agentes comunitários. Além destes, há profissionais de saúde bucal (dentista e auxiliar de consultório dentário) que fazendo parte das duas equipes. Cada equipe é responsável por 6 microáreas dentro do território.

\subsection{Espaço para planejamento e ações conjuntas}

As equipes não possuem uma coordenação fixa, deste modo, as decisões são compartilhadas pelos profissionais em conjunto. Os grupos, que eram realizados antes da pandemia, assim como as ações do Programa Saúde na Escola, visitas domiciliares são realizadas junto com todos da equipe, ou então planejados juntos com todos os membros.

Acerca das ações realizadas pela equipe, sendo elas juntas ou separadas, o trabalho mesmo que realizado individualmente, como em uma consulta de pré-natal, carrega informações que perpassam pelo atendimento odontológico, na sala de vacinas, e na interconsulta médica, o que demonstra a importância do trabalho multidisciplinar. As ações do núcleo de cada profissional, como consultas de enfermagem, médica e da odontologia, são realizadas separadamente. Os efeitos do trabalho multiprofissional são percebidos através do processo cotidiano, no qual a responsabilidade do desempenho é de cada profissional. Por outro lado, o trabalho interdisciplinar é percebido claramente nos casos que envolvem as doenças crônicas e gestantes.

$\mathrm{Na}$ organização interna das equipes, as discussões e debates ocorrem sempre entre ou após um atendimento, podendo ser também em reuniões com a equipe, as quais ocorrem semanalmente. Porém, não há um planejamento para que as interações do trabalho em equipe ocorram de modo organizado, somente surgem estas discussões nas reuniões fechadas

Comumente não se transcorre um momento de sensibilização para o trabalho integrado no serviço. Entretanto, é percebido que as reuniões de equipes são ferramentas utilizadas de uma maneira empírica para que ocorra o trabalho em equipe. Sendo este, um espaço que a maioria dos profissionais expõe seus encaminhamentos e sugestões para os casos e processos de trabalho.

O debate sobre trabalho interprofissional precisa vir acompanhado de um movimento de reconhecimento e valorização de todos os profissionais. Enquanto as relações de poder e as formas de manutenção da desigualdade de poder não forem enfrentadas nas esferas macro, meso e microssocial, 


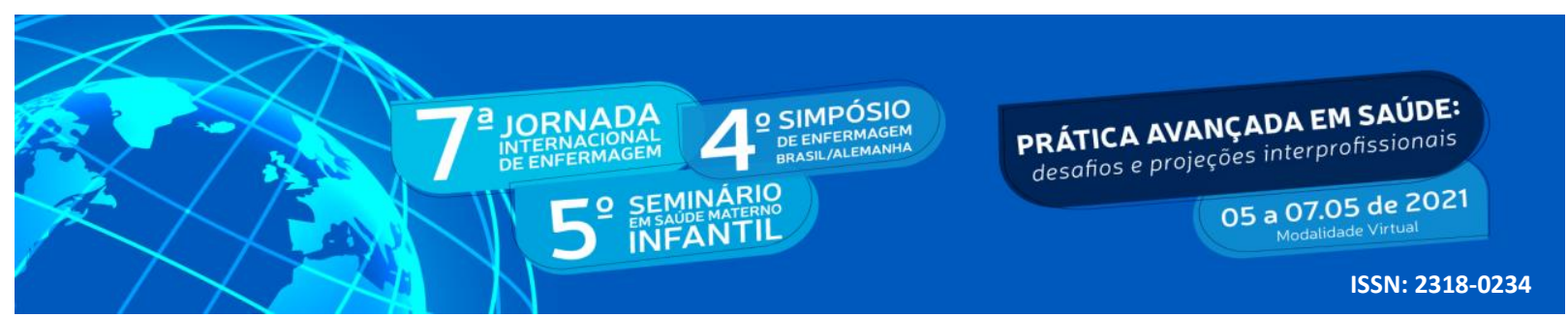

teremos dificuldades de avançar em uma cultura colaborativa (BARROS; SPADACIO; COSTA, 2018, p. 169).

Ressalta-se que as reuniões são espaços em que cada profissional se posiciona a partir da sua especialidade e ocorre a discussão sobre determinadas condutas entre todos os profissionais. De tal modo, é o espaço mais utilizado para discutir e refletir sobre os mesmos objetivos.

\subsection{Comunicação entre profissionais}

Dentre os desafios na comunicação entre os profissionais da equipe, percebe-se falhas na comunicação ou má interpretação dos diálogos, gerando assim uma insatisfação, podendo refletir em comunicação violenta ou entendimento dúbio. É considerado que a relutância de alguns profissionais em trabalhar em equipe está relacionada com a facilidade de trabalhar somente em seu núcleo, pois torna-se mais prático e fácil de ser conduzido. Contudo, é necessário perceber quando isso acontece.

A formação acadêmica propõe estudos e atividades em prol do conhecimento e exercício do trabalho em equipe, e salienta a importância deste no atendimento ao público. O trabalho multidisciplinar é visto com aquele desempenhado por cada núcleo, contudo sem a sua integração em equipe. Já a interdisciplinaridade pressupõe a colaboração de vários núcleos que se relacionam entre si.

A multiprofissionalidade e a interprofissionalidade se referem à prática profissional. A primeira pressupõe que diferentes profissões estejam atuando juntas, mas não necessariamente havendo interação ou um propósito claro. Do contrário, a interprofissionalidade implica na interação entre as profissões com vistas à colaboração em torno de um objetivo comum. (ELY; TOASSI, 2017, p. 83)

Por fim, na narrativa apresentada emerge a existência de certa resistência diante do trabalho em equipe. Visto que, o trabalho multiprofissional carece de um alinhamento das ações por meio da comunicação e o fazer juntos, com o devido apoio e suporte de todos.

Ademais, a qualidade do trabalho é afetada diretamente pelos desafios que a equipe enfrenta em seu cotidiano, como o número de usuários majoritariamente do que as recomendadas pelo ministério da saúde, muitas_microáreas sem agentes de saúde e a falta de médicos de 40h. Porém, o trabalho em equipe traz muitos benefícios para o funcionamento da unidade, integrando as diferentes visões de cada profissional de saúde sobre o que está sendo 


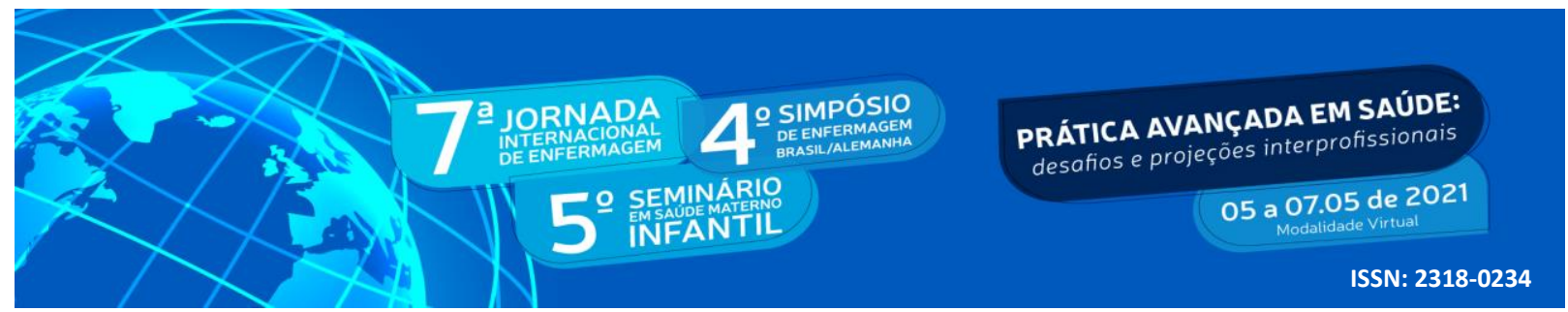

discutido, além do conhecimento maior dos agentes comunitários sobre o funcionamento familiar da comunidade.

\section{CONCLUSÃO}

A percepção da enfermagem quanto a equipe multiprofissional compreende que mesmo atuando de forma coesa e sistematizada, ainda assim, os desafios e dificuldades podem permear ao longo do percurso. Portanto, nas relações entre individual e coletivo, universal e singular, não devemos escolher apenas um dos lados desses aparentes opostos, mas passar a vê-los como partes intercambiantes.

A equipe multiprofissional mostra-se importante para a humanização e resolutividade dos serviços do Sistema Único de Saúde, mas existem paradigmas a serem superados. É necessário enfrentar a interação entre esses elementos distintos, mas igualmente constitutivos de uma mesma realidade, sabendo delinear situações da qual possam intervir de forma equivocada na atuação em grupo.

Para fortalecer os espaços para a efetivação do trabalho conjunto das equipes, que podem beneficiar tanto o serviço de saúde em que é ofertado atendimento aos usuários quanto a própria equipe, fortificando o embasamento e compartilhamento de saberes em prol de uma mesma causa. E, assim, proporcionar uma maior resolutividade do serviço, ao mesmo tempo, estimulando o vínculo da equipe no que corresponde a sua complexidade de atuar.

Apesar do trabalho em equipe exigir de cada profissional um conhecimento específico, busca-se ressaltar que esta ação poderá ser pouco eficaz quando as práticas dos mesmos atuarem somente focados em seus saberes de forma isolada. Ou seja, o conhecimento não é válido quando não compartilhado em equipe, os ofícios a serem exercidos serão somente condutas condicionadas pela facilidade da busca em realizar somente o que lhe convém. Por este motivo é necessário a comunicação uns com os outros, e assim desmoronar as causalidades lineares e as fronteiras territoriais demarcadas pelos excessos disciplinares.

\section{REFERÊNCIAS}

ANJOS FILHO, N. C.; SOUZA, A. M. P.. A percepção sobre o trabalho em equipe multiprofissional dos trabalhadores de um Centro de Atenção Psicossocial em Salvador, Bahia, Brasil. Interface (Botucatu), Botucatu , v. 21, n. 60, p. 63-76, Mar. 2017 . Disponível em: 


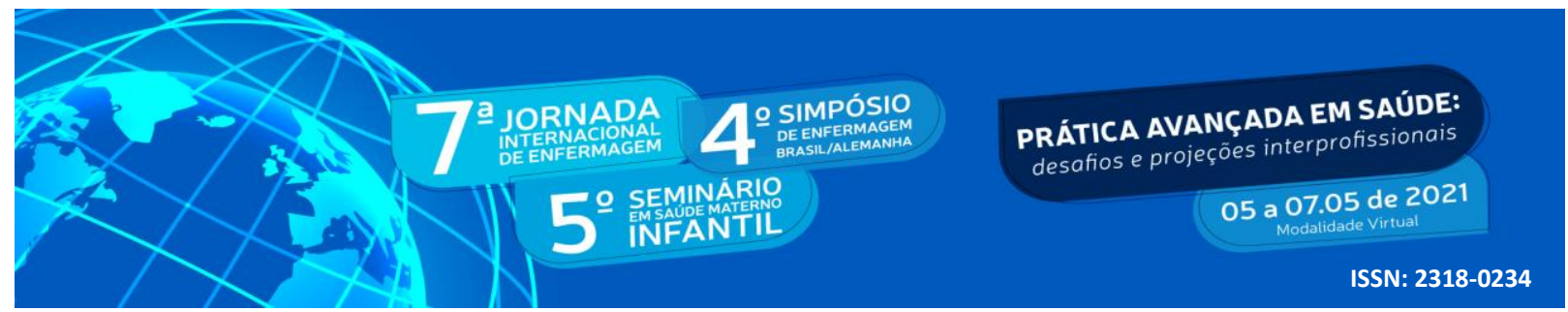

http://www.scielo.br/scielo.php?script=sci_arttext\&pid=S1414-

32832017000100063\&lng=en\&nrm=iso. Acesso em: 18 dez. 2020.

ALVES, A. K. D. S; SANTOS, J. M. S. D; LOPES, R. F.. O papel da enfermagem na equipe multiprofissional no contexto da atenção primária: revisão integrativa de literatura. Gep News, Maceió, v. 2, n. 2, p. 359-366, abr./2019. Disponível em: <https://www.seer.ufal.br/index.php/gepnews/article/view/7923>. Acesso em: 18 dez. 2020.

BACKES, S.D. et al. Trabalho em equipe multiprofissional na saúde: da concepção ao desafio do fazer na prática. Rev. Eletrônica Disciplinarum Scientia. v. 15, n. 2, 2014. Disponível em: https://periodicos.ufn.edu.br/index.php/disciplinarumS/article/view/1093. Acesso em: 19 Dez. 2020.

BARROS, N. F., SPADACIO, C. e COSTA, M. V. Trabalho interprofissional e as Práticas Integrativas e Complementares no contexto da Atenção Primária à Saúde: potenciais e desafios. Saúde em Debate [online]. 2018, v. 42, n. spe1, pp. 163-173. Disponível em: <https://doi.org/10.1590/0103-11042018S111>. Acesso em: 18 dez. 2020.

BRASIL. Ministério da Saúde. Conselho Nacional de Súde. Resolução nº 510, de 7 de Abril de 2016. Ministério da Saúde, Brasília, DF., 7 abr. 2016.

CRUZ, F. E. B. D; ARAÚJO, A. H. I. M. D; BEZERRA, M. L. R. Liderança de enfermagem na saúde pública: revisão integrativa da literatura. Revista JRG de Estudos Acadêmicos, São Paulo, v. 3, n. 07, p. 577-584, dez./2020. Disponível em: http://www.revistajrg.com/index.php/jrg/article/view/84. Acesso em: 19 dez. 2020.

DALTRO, M. R.; FARIA, A. A. D. Relato de experiência: Uma narrativa científica na pósmodernidade. Estudos e Pesquisas em Psicologia, Rio de Janeiro, v. 19, n. 1, 2019. Disponível em: https://www.e-publicacoes.uerj.br/index.php/revispsi/article/view/43015/29664. Acesso em: 19 dez. 2020.

ELY, L. I. , TOASSI, R. F. C. Atividade de ensino integradora dos currículos na graduação em saúde: entre o multiprofissional, o interdisciplinar e o interprofissional. In: TOASSI, Ramona Fernanda Ceriotti. Interprofissionalidade e formação na saúde : onde estamos? 1. ed. Porto Alegre: Rede UNIDA, 2017. p. 81-97. Disponível em: https://lume.ufrgs.br/handle/10183/183996. Acesso em: 19 dez. 2020.

LANCA, T. A.; AMARAL, R. M.; GRACIOSO, L. S. Multi e interdisciplinaridade nos programas de pós-graduação em Ciência da Informação brasileiros. Perspect. ciênc. inf., Belo Horizonte, v. 23, n. 4, p. 150-183, Dez. 2018.

PEDUZZI, M.; AGRELI, H. F.. Trabalho em equipe e prática colaborativa na Atenção Primária à Saúde. Interface - Comunic., Saúde, Educ. [online]. v. 22, n. Suppl 2, 2018. Disponível em: https://doi.org/10.1590/1807-57622017.0827.Acesso em: 19 dez. 2020 


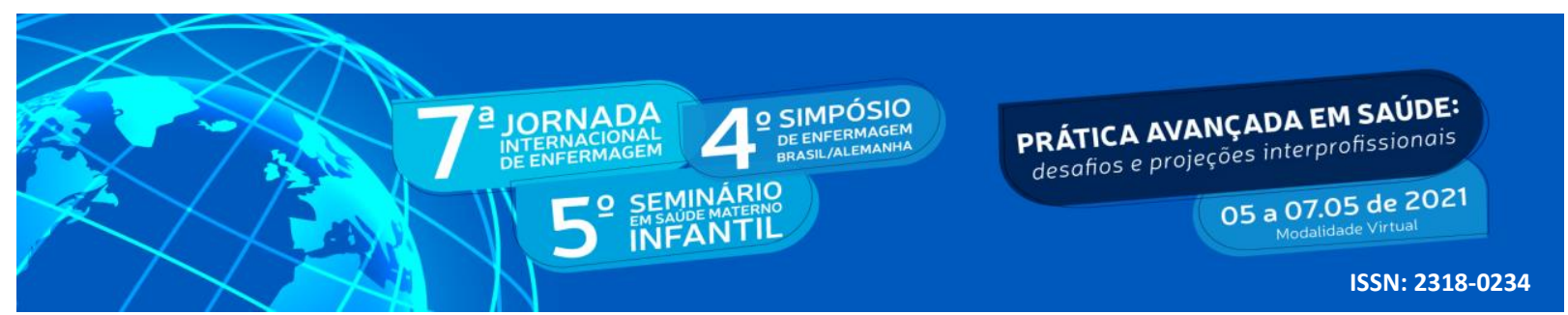

